\title{
Erratum: Quantum Hydrodynamics of a Bose Fluid: ac Josephson Effect in Superfluid Helium*
}

\author{
A. C. Biswas \\ Tata Institute of Fundamental Research. Bombay, India
}

(Received March 18, 1975)

The expression for the Josephson current, Eq. (32), should be replaced by

$$
j_{A B}=\mathbf{q} \cdot(\hbar / m) \nabla \rho_{A} \sin \left(\mu_{A B} / \hbar\right) t
$$

where $\rho$ is the mass density at $A$. The temperature range at which this current should be observable is given by $\lambda_{T} \gg \xi_{c}$, where $\lambda_{T}$ is the thermal wavelength and $\xi_{c}$ is the coherence length. Both the Ginzburg-Pitaevskii [Sov. Phys.-JETP 7, 858 (1958)] and Mamaladze [Sov. Phys.-JETP 25, $479(1967)]$ estimates of $\xi_{c}$ give the temperature $T$ as $T \ll 1 \mathrm{~K}$.

J. Low Temp. Phys. 18(1/2), 3 (1975). 